\title{
Exposure to Asbestos and Mesothelioma of the Pleura
}

\author{
P. B. S. FOWLER,* D.M., F.R.C.P. ; J. C. SLOPER, † M.D., M.R.C.P. ; E. C. WARNER,* M.D., F.R.C.P.
}

Brit. med. F., 1964, 2, 211-213

Two cases of mesothelioma were the subjects of fairly recent coroners' inquests. In neither instance was it accepted that the tumour was attributable to inhalation of asbestos fibre; yet in one case there was a known and severe occupational history of exposure to asbestos, while in both cases "asbestos bodies" were found in the lung. A point made at both inquests was the difficulty of attributing a tumour to asbestos-inhalation in the absence of the diffuse pulmonary fibrosis so long recognized (Murray, 1907) as characteristic of " asbestosis."

Recently, however, it has become clear, at least in South Africa (Wagner, Sleggs, and Marchand, 1960 ; Thomson, Kaschula, and MacDonald, 1963), that the association between mesotheliomata and asbestos-inhalation can scarcely be fortuitous. The studies initiated by Wagner in Great Britain (1963, unpublished) suggest that the same may apply here. Our two cases of this rare tumour illustrate the practical implications of his investigations.

\section{Case 1}

A man born in 1902 was investigated in Charing Cross Hospital in 1957 for mild hypertension. During a routine medical examination he denied shortness of breath, cough, or other symptoms, and a chest $x$-ray film, even when studied retrospectively, showed no evidence of lung disease. He was somewhat polycythaemic (Hb $122 \%-18.1$ g./100 ml.; R.B.C. 5,870,000; packed cell volume $53 \%$ ).

Over the next two years he lost weight and complained of abdominal distension after meals. In February 1961 he became

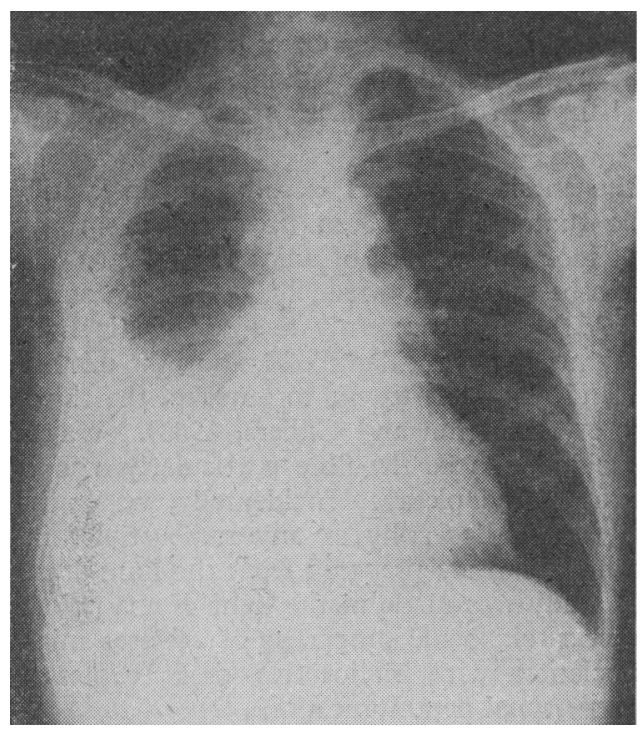

FIG. 1.- Radiograph of chest in Case 1. The nodular opacities on the mediastinal and latera surfaces of the right pleura suggest a mesothelioma.

short of breath on exertion and had a slight cough with scanty sputum. In May he had developed the signs of a large right-sided pleural effusion and was again admitted. By this time he was. plethoric but wasted. There was no clubbing. The rest of the

* Physician, Charing Cross Hospital.

† Honorary Consultant Pathologist, Charing Cross Hospital. clinical examination was essentially negative, except for an anaemia ( $\mathrm{Hb} 78 \%$ ). His chest was aspirated and two pints $(1.141$.$) of$ slightly blood-stained fluid was withdrawn. No malignant cells were seen and culture was sterile. Radiographs at this stage showed a very thickened pleura over the whole of the right chest, with a nodular outline (Fig. 1) which suggested a mesothelioma ; on biopsy an anaplastic growth was found. Despite the absence of evidence of fibrosis in the contralateral lung, and of any fibrosis in the chest radiograph of 1957, the possibility of asbestos-inhalation was considered. No asbestos bodies were found in the sputum, but questioning revealed that this patient had cleaned bags in an asbestos factory from 1926 to 1929, and had continued with the same firm, partly as a cleaner, partly as a canteen attendant, until 1942 . For the next 20 years he was not exposed to asbestos. Despite treatment involving the insertion of radioactive gold into the pleural cavity, his condition deteriorated and he died at home.

\section{Necropsy}

This was performed for H.M. Coroner by Dr. B. H. Knight at Farnborough Hospital, Kent, the right lung and diaphragm being given to us for examination.

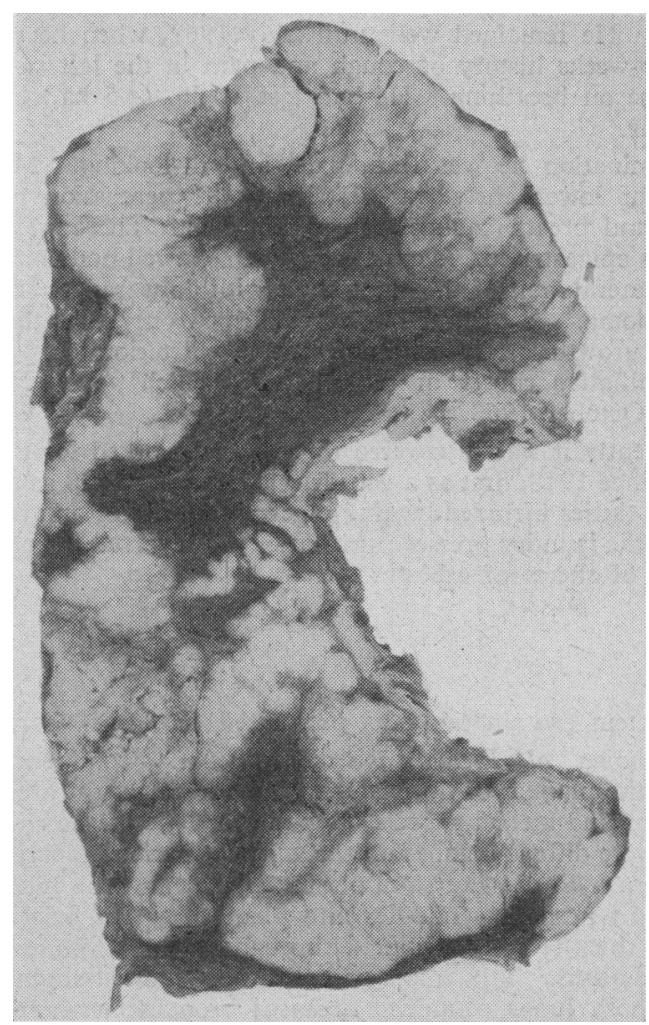

Fig. 2.-Photograph of slice of right lung from Case 1 to show cuirass-like sheet of pleural growth.

Mesothelioma of Pleura.-It was noted that the tumour was confined to the right side of the chest and did not penetrate the diaphragm. There was no growth in the left lung or elsewhere in the body. The right lung was largely enveloped by a shell of firm white growth (Fig. 2) varying in general between 0.5 and $1.7 \mathrm{~cm}$. in thickness, but thickened up to $8 \mathrm{~cm}$. On its lower surface, which included the diaphragm. In several areas the growth was 
soft and necrotic. Elsewhere growth bulged into the substance of the lung, but nowhere were there deep islands of growth within the lung, nor, despite prolonged search, could a bronchial primary carcinoma be found. Four anthracotic glands were found at the hilum, and also a white ovoid mass $(2 \times 1 \mathrm{~cm}$.) which proved to be a neurofibroma.

\section{Microscopy}

Smears from the unfixed lung revealed asbestos bodies. These were found only with great difficulty in thin $(5-\mu)$ paraffin sections, but were readily demonstrated in thick $(30-\mu)$ frozen sections. Typically, they measured $150 \mu$ in length, and lay in loose fibrous tissue. They stained intensely for haemosiderin with Perls's Prussianblue technique.

These sections of lung revealed focal collapse of alveoli with some increase in interstitial collagen; whether this reflected the condensation of stroma or a slight focal fibrosis could not be determined. Some bronchioles contained purulent exudate, but evidence of chronic bronchitis-for example, the loss of submucosal elasticawas lacking. Arterioles showed fibroelastic intimal hyperplasia.

The mesothelioma was composed in some areas of sheets and trabeculae of cuboidal and polygonal cells. In places these cells lined clefts, in places they were papillary in arrangement. In many areas these cells were set in a diffuse and densely collagenous stroma. This tumour was stained fairly intensely by Alcian blue. It contained no asbestos bodies. There was growth in one of the hilar glands, which also contained asbestos bodies.

\section{Case 2}

A man born in 1889 was first seen in March 1961, when he presented with the typical symptoms and signs of temporal arteritis. This was confirmed by biopsy (see below), which also relieved the symptoms. He remained well until May 1962, when he presented with a two-weeks history of cough and pain in the left side of the chest, worse on breathing. He had lost $10 \mathrm{lb}$. $(4.5 \mathrm{~kg}$.) in weight in one year.

On examination he was thin, pale, and ill-looking. Movement of the right lower chest was restricted, the percussion note was impaired, and breath sounds were diminished. There was a large mass in the epigastrium. A chest $x$-ray film showed nodular shadows along the lateral wall of the right chest with appearances suggesting a mesothelioma. A pleural biopsy confirmed only that this was a malignant growth. His condition steadily deteriorated. $\mathrm{He}$ was given a palliative course of radiotherapy, which gave relief. $\mathrm{He}$ died on 2 October 1962.

Subsequently it was discovered that he had worked for a laundry company since 1918, first as a van-driver, but from 1938 as a general hand. His duties included lagging pipes with asbestos and the maintenance of the laundry presses ; this latter job involved the occasional reinsertion of sheets of asbestos.

\section{Necropsy}

Post-mortem examination was performed for H.M. Coroner.

Mesothelioma of Pleura and Peritoneum.-Confluent, partly necrotic white plaques of fibrous growth up to $5 \mathrm{~cm}$. thick enveloped the posterior and medial aspects of the lower lobe of the right lung, and there were diffuse plaques on the adjacent parietal pleura. There was no growth in the deep lung parenchyma, and the hilar glands were spared; numerous nodules and plaques (up to $2.5 \times 2$ by up to $0.3 \mathrm{~cm}$. thick) were distributed throughout the peritoneal cavity, sparing the pelvis. The apices of both lungs showed slight emphysema and both lungs contained areas of bronchopneumonia. The right lung, compressed by pleural growth, showed areas of partial collapse. There were occasional fibrous pleural adhesions over both lungs, but the pleura was not markedly thickened, save where involved by the fibrous growth.

\section{Microscopy}

Smears from the right lung revealed asbestos bodies. Autoradiographs prepared from such bodies and exposed for 42 days revealed no local radioactivity. Asbestos bodies were found with great diffi- culty in thin $(5-\mu)$ paraffin sections, but readily in thick $(30-\mu)$ frozen sections. These bodies lay either in alveolar spaces or in areas of slight interstitial fibrosis; they were not found in many sections taken from the pleural growth. Paraffin and frozen sections of a hilar gland did not reveal asbestos bodies. There were areas of slight interstitial fibrosis, the latter especially in the partly collapsed right lower lobe.

The 'mesothelioma was very similar to that in Case 1, and in places penetrated into the lung through the pleural elastica. It stained fairly intensely with Alcian blue, the reacting material being removed by hyaluronidase.

Other findings at necropsy included bronchopneumonia, a thrombotic embolus in a pulmonary artery, and partial occlusion of the left renal vein by thrombus. A biopsy of the temporal artery (1961 ; courtesy of Dr. Jones Williams, Mount Vernon Hospital, Middlesex) revealed changes consistent with temporal arteritis; the single paraffin section we examined was devoid of asbestos bodies.

\section{Discussion}

Pleural mesotheliomata, primarily on account of their remarkable appearance both on radioscopy and also at necropsy, have come to be regarded by many (McCaughey, 1958; Wagner, Sleggs, and Marchand, 1960) as a clinical and pathological entity distinct from carcinoma of the bronchus. In both our cases the manner in which growth enveloped and compressed the lung led to the radioscopic diagnosis of mesothelioma ; and at necropsy the cuirass-like shell of pleural growth was quite unlike the usual appearance of the carcinoma of the bronchus. Detailed examination of the lung failed to reveal a primary bronchial carcinoma, although, as in all such cases, it must be admitted that such a primary could be missed (Willis, 1948 ; Smart and Hinson, 1957). Microscopy is of less value in distinguishing mesotheliomata, for these tumours are anaplastic and vary in their histological appearance, forming in some areas large amounts of dense collagenous tissue containing clefts lined by cubical and columnar epithelium, whereas in other areas they are composed of sheets of polygonal undifferentiated cells (McCaughey, 1958). Wagner, Munday, and Harington (1962) claim that such tumours, when fixed in formol-alcohol-acetic mixtures, secrete material which stains as acid mucopolysaccharide, a feature which helps in their histological differentiation from peripheral pulmonary adenocarcinomata. Our cytochemical observations, because they are made on material fixed in formol-saline, are of limited value in this respect.

The pathogenesis of pleural mesotheliomata is still obscure. Comparable tumours have been produced in mice after the intrapleural inoculation both of silica and of two of the three varieties of asbestos in use-namely, crocidolite and chrysotile (Wagner, 1962). More recently a carcinogenic hydrocarbon, 3:4-benzpyrene, has been identified among the oils adsorbed to crocidolite and, to a less extent to amosite, the third form of asbestos fibre (Harington and Cilliers, 1963). Wagner (1963) believes crocidolite to be the fibre particularly associated with mesothelioma of the pleura. Incidentally, we could find no evidence by autoradiography of any radioactivity in asbestos bodies present in smears prepared from the lung of our second case. This study was made in the light of the possibility that the supposed carcinogenic properties of asbestos might be due to its association with radioactive ores (Professor A. G. Heppleston, personal communication).

Indirect evidence linking asbestos fibres with the development of mesotheliomata can be sought by determining whether the association noted in human patients is fortuitous or not. It is necessary to know the incidence of mesotheliomata in the general population, the extent to which the general population is exposed to asbestos fibres, and the proportion of cases of mesothelioma of the pleura similarly exposed.

The disease is clearly much rarer than, for example, primary carcinoma of the bronchus. Thus only one of the 18 instances 
of lung carcinoma reported by Doll (1955) in patients known to have been severely exposed to asbestos was described as a mesothelioma ("endothelioma"). Again, Saccone and Coblenz (1943) calculated the incidence of this tumour as 1 per 1,100 necropsies in the U.S.A.; but comparable figures for this country are lacking.

Little is known, too, about the risk of the inhalation of asbestos fibres here. In this respect specific information concerning air-pollution to windward and leeward of asbestos factories would be of value. More immediate evidence, however, is likely to be derived from the study of sputum in the general population, and particularly of lung smears made in large series of necropsies. By this latter method Thomson et al. (1963) found evidence of exposure in over $25 \%$ of 500 necropsies made in an urban area in South Africa. They also mentioned that high figures had been obtained in Miami, Florida. Although their findings were not checked by the microchemical identification of asbestos bodies in affected lungs-a step which, where possible, should be attempted (see Gloyne, 1951 ; Gough, 1959)-the implied proposition seems reasonable, namely that the histological identification of asbestos bodies provides a guide to the incidence of exposure to asbestos. South Africa, however, is a primary producer of asbestos, and it seems unlikely that exposure in Great Britain is as high.

With regard to the presence of asbestos bodies in the lungs of patients suffering from mesotheliomata, this was given little attention by those investigating these tumours until Wagner and his co-workers in South Africa (1960) reported their presence in 8 out of the 10 cases of pleural mesothelioma they examined, in which lung tissue as well as tumour was available for examination. It seems to us that the figures could not be accounted for by the chance association between exposure to asbestos and the development of mesotheliomata, even if one in four of the general population were exposed to asbestos $(\mathbf{P}=$ 0.0003 ). Indeed, even if one in ten of the population in Great Britain were shown to have asbestos bodies in their lungs, the presence of such bodies in both our cases could scarcely be fortuitous $(P=0.01)$. Our evidence, however, will be more convincing when the larger series to which Wagner (1963) has referred has been published.

Meantime it must be stressed that asbestos bodies should be sought in the lungs of all patients with mesotheliomata: this search should be pursued even though there is no occupational history of exposure to asbestos and even though there is no evidence of severe pulmonary fibrosis.

In searching for these bodies it is suggested, first, that smears be prepared from the fresh cut surface of the lung, as recommended, for example, by Thomson et al. (1963). Second, in those units which lack the facilities necessary for the preparation of thick whole-lung sections, we suggest that small thick $(30-\mu)$ frozen sections of lung be prepared and treated with Perls's potassium ferricyanide technique. Asbestos bodies can then readily be recognized by their shape and content of haemosiderin. If thin $(5-\mu)$ paraffin sections are used, rigid asbestos bodies, measuring some $150 \mu$ in length, must often be torn out and lost.

\section{Summary}

Two cases of mesothelioma of the pleura are reported.

Despite the absence of severe pulmonary fibrosis, the lungs in both contained ",asbestos bodies"; in both, too, there was an occupational history of exposure to asbestos, although in the second this was obtained with difficulty and after death.

The association between mesothelioma of the pleura and exposure to asbestos is unlikely to be fortuitous; but to establish this point all cases of mesothelioma, whatever their occupational history, should be studied with this possibility in mind; for evidence is now accumulating that this disease is an industrial hazard.

ADDENDUM.- Since the submission of this paper an analysis of the material collected in the Bernhard Baron Institute of Pathology of the London Hospital between 1917 and 1962 has confirmed the frequency with which asbestos fibres occur in the lungs of patients with mesotheliomata (Hourihane, 1964).

Particular thanks are due to Dr. Gavin Thurston, Queen's Coroner for Westminster, for his comments, and Dr. B. N. Knight for his help with the first case.

\section{REFERENCES}

Doll, R. (1955). Brit. F. industr. Med., 12, 81.

Gloyne, S. R. (1951). Lancet, 1, 810.

Gough, J. (1959). "Occupational Pulmonary Diseases." In Modern Trends in Pathology, edited by D. H. Collins, p. 296. Butterworth, London.

Harington, J. S., and Cilliers, J. J. le R. (1963). Geochim. et cosmoch. Acta, 87, 411 .

Hourihane, D. O’B. (1964). Thorax, 19, 268.

McCaughey, W. T. E. (1958). 7. Path. Bact., 76, 517.

Murray, H. M. (1907). Report of the Departmental Committee on Compensation for Industrial Disease, para. 4176-4104. H.M.S.O., London.

Saccone, A., and Coblenz, A. (1943). Amer. F. clin. Path., 13, 186.

Smart, J., and Hinson, K. F. W. (1957). Brit. F. Tuberc., 51, 319.

Thomson, J. G., Kaschula, R. O., and MacDonald, R. R.'(1963). S. Afr. med. $¥ ., 37,77$.

Wagner, J. C. (1962). Nature (Lond.), 196, 180.

- (1963). Author's unpublished summary of paper read at Fourteenth International Congress on Occupational Health, Madrid, Sept., 1963. International Congress on Occupational Health, Madrid, Sept., 1963. Sleggs, C. A., and Marchand, P. (1960). Brit. F. industr. Med.,

17, 260. (1948). Pathology of Lung Tumours, 1st ed., pp. 184, 185.
Willis, R. A. Butterworth, London. 